\title{
ยกி

\section{Acessibilidade em objetos de aprendizagem na EaD: uma Análise em um Curso de Especialização}

\section{Juliana Dalbem Omodei ${ }^{1}$, Edison Trombeta de Oliveira², Marcos Barros de Souza $^{3,}$ Vanderlei Leite dos Santos ${ }^{4}$}

\footnotetext{
1 Doutoranda em Educação, Faculdade de Ciências e Tecnologia, Universidade Estadual Paulista (Unesp/FCT). Rua Roberto Simonsen, 305 - Centro Educacional - Presidente Prudente - SP - Brasil. judalbem@gmail.com

2 Doutorando em Educação, Faculdade de Educação, Universidade de São Paulo (USP). Avenida da Universidade, 308, São Paulo - SP - Brasil. edisontrombeta@gmail.com

3 Pesquisador, Faculdade de Ciências e Tecnologia, Universidade Estadual Paulista (Unesp/FCT). Rua Roberto Simonsen, 305 - Centro Educacional - Presidente Prudente - SP - Brasil. souzamb@bol.com.br

4 Professor especialista, Universidade Paulista de São Paulo (Unip). Avenida Vergueiro, 4300, São Paulo SP. vandersantos@ig.com.br
}

\section{Resumo}

Este trabalho tem como objetivo analisar os objetos de aprendizagem oferecidos em duas disciplinas do curso de especialização em Educação Especial na perspectiva da Educação Inclusiva, sob a ótica da acessibilidade. Os objetos de aprendizagem são recursos digitais reutilizáveis e que auxiliam a aprendizagem de algum conceito. Para atingir o objetivo proposto, foi empreendida uma pesquisa bibliográfica seguida de um estudo de caso. Para a coleta de dados optou-se no estabelecimento de categorias de análise que permitiram obter uma visão global dos recursos. Os dados foram analisados qualitativamente etapa a etapa de cada jogo, e os dados coletados foram organizados em uma planilha previamente elaborada para esse fim. Os resultados evidenciaram que, embora haja esforço da universidade promotora do curso em oferecer acessibilidade em todo o ambiente virtual e materiais disponíveis, ainda são necessárias algumas adaptações nos objetos de aprendizagem para que se tornem totalmente acessíveis às pessoas com necessidades especiais em nível sensorial. Todavia, é por meio de iniciativas como essa que são possíveis o crescimento e o aprimoramento de objetos educacionais acessíveis e, dessa forma, contribuir com o acesso de permanência de todos na educação a distância.

Palavras-chave: Objetos de aprendizagem; Acessibilidade; Educação a Distância. 


\title{
Accessibility in Objects Learning in EAD: an Analysis on a Course of Expertise
}

\begin{abstract}
This work aims to analyze the learning objects offered in two course subjects of specialization in Special Education from the perspective of inclusive education from the perspective of accessibility. The learning objects are reusable digital resources and that help learning some concept. To achieve this purpose a literature search followed by a case study was undertaken. To collect data it was decided to establish categories of analysis, which provided an overview of resources. Data were analyzed qualitatively, step by step each game, and collected data organized in a spreadsheet previously prepared for this purpose. The results showed that although there is a university effort to offer accessibility across the virtual environment and available materials are still needed some adjustments to the learning object to become fully accessible to people with sensory disabilities. However, it is through such initiatives that are possible growth and improvement of accessible educational materials and thus contribute to the permanence of access for all in the distance.
\end{abstract}

Keywords: Learning objects; Accessibility; Distance education. 


\section{Introdução}

O conceito de objeto de aprendizagem (OA) não é consensual e sua definição pode variar de acordo com a abordagem proposta. Para Audino e Nascimento (2010, p. 130), os OAs são "materiais importantes no processo de ensino e aprendizagem, pois nos fornecem a capacidade de simular e animar fenômenos, entre outras características, assim como reutilizá-los em vários outros ambientes de aprendizagem".

De acordo com Beck (2001, apud Wiley, 2002, p. 1) os OAs

são considerados como qualquer recurso digital que possa ser reutilizado para o suporte ao ensino. A principal ideia dos objetos de aprendizado é quebrar o conteúdo educacional em pequenos pedaços que possam ser reutilizados em diferentes ambientes de aprendizagem, em um espírito de programação orientada a objetos.

Na definição de Spinelli (s/d, p. 7),

um objeto virtual de aprendizagem é um recurso digital reutilizável que auxilie na aprendizagem de algum conceito e, ao mesmo tempo, estimule o desenvolvimento de capacidades pessoais, como por exemplo imaginação e criatividade. Dessa forma, um objeto virtual de aprendizagem pode tanto contemplar um único conceito quanto englobar todo o corpo de uma teoria. Pode ainda compor um recurso didático, envolvendo um conjunto de atividades, focalizando apenas determinado aspecto do conteúdo envolvido, ou formando, com exclusividade, a metodologia adotada para determinado trabalho.

Wiley (2001, p. 3) define que "os objetos de aprendizagem são elementos de um novo tipo de instrução baseada em computador apoiada no paradigma da orientação a objetos da informática. A orientação a objetos valoriza a criação de componentes (chamados "objetos") que podem ser reutilizados em múltiplos contextos".

Audino e Nascimento (2010, p. 134, apud Singh, 2001) apontam que os OAs devem ser "estruturados e definidos em três partes bem definidas, pois essa estrutura diferencia o recurso de outras tecnologias aplicadas à educação e possibilita a produção de conhecimento". São eles:

1. Objetivos: sua finalidade é demonstrar ao aluno o que pode ser aprendido a partir do estudo desse objeto de aprendizagem, além dos conceitos necessários para um bom aproveitamento do conteúdo. 
2. Conteúdo instrucional ou pedagógico: parte que apresenta todo o conteúdo necessário para que, ao término, o aluno possa atingir os objetivos definidos.

3. Prática e feedback: a cada final de utilização, julga-se necessário que o aluno registre a interação com o objeto para a produção de conhecimento; isto é, confirma-se se as hipóteses ou opções do aluno estão corretas ou são dadas orientações para ele ir continuar buscando novas respostas.

Assim, quando esses objetos são utilizados na Educação a Distância o processo de ensino-aprendizagem pode ser potencializado de maneira lúdica, divertida e interativa.

O principal objetivo de um OA é promover a reflexão crítica do estudante sobre o conteúdo estudado, despertar a curiosidade, o questionamento, a inquietação. Sua utilização na Educação a Distância evita a padronização da atividade, avaliação e feedback automatizados e valoriza as experiências do aluno. Dessa forma, em um curso a distância o conteúdo educacional não se limitaria à transmissão das informações, mas a uma aprendizagem ativa, por meio da interação com o conteúdo de forma prática. Tais aspectos estão de acordo com os autores Sampaio e Almeida (2010), que afirmam que o uso do OA interativo também atenderia ao ideal de equidade na educação e, especificamente no desafio da acessibilidade, ampararia os aprendizes dotados de necessidades especiais (Rehak \& Mason, 2003, apud Sabbatini, 2012).

O tema acessibilidade tem tomado força especialmente após a Declaração de Salamanca - 1994 (Unesco, 1998), que pretendeu assegurar a educação das pessoas com necessidades especiais como parte do sistema educacional. Aliado a isso, o Artigo 26 da Declaração Universal dos Direitos Humanos dispõe que o acesso aos estudos superiores deve estar aberto a todos em plena igualdade. Considerando que a Educação a Distância é uma modalidade de ensino que tem crescido vertiginosamente nos últimos anos, deve contemplar o acesso a todas as pessoas sem qualquer distinção. Sua expansão pode contribuir com a democratização do ensino, principalmente quando permite o acesso ao Ensino Superior, seja na graduação ou pós-graduação, às pessoas com algum tipo de necessidades especiais. 
Assim, conteúdos, mídias e tecnologias relativos à Educação a Distância também devem se adequar, de forma a oferecer acessibilidade a essas pessoas, rompendo as barreiras de acesso aos níveis superiores de educação.

Diante desse contexto, a acessibilidade em OA deve ser implementada desde seu projeto de criação - e não após sua criação -, com adaptações às necessidades dos alunos. Pesquisas realizadas por Macedo (2013) detectaram extensa quantidade de recomendações que focalizam acessibilidade em OA. Entretanto, tais recomendações estão voltadas para analistas, programadores e webdesigners implementadores de material didático.

Segundo as diretrizes para a criação de objetos de aprendizagem acessíveis,

todo conteúdo de um objeto de aprendizagem deve apresentar pelo menos:

- Uma mídia equivalente, em formato diferente ou uma mídia alternativa se não for possível uma mídia equivalente;

- Uma mídia de acesso textual, equivalente ou alternativo (Macedo, 2013, p. 129).

Uma mídia equivalente é aquela que apresenta um conteúdo idêntico à outra, porém em uma modalidade diferente. Por exemplo, um texto disponível em áudio e o mesmo texto associado a um arquivo para impressão em Braille. O conteúdo alternativo é uma ampliação do conteúdo equivalente e é fornecido de forma diferente, porém com o mesmo objetivo final de aprendizagem (Macedo, 2013).

Assim, o desenvolvimento de objetos de aprendizagem acessíveis se justifica pela necessidade de proporcionar igualdade de acesso e permanência em cursos a distância a todas as pessoas, independentemente de suas limitações.

\section{Metodologia}

\subsection{O campo de pesquisa}

O presente estudo teve como campo de pesquisa o Curso de Especialização em Educação Especial na Perspectiva da Educação Inclusiva. O curso é fruto de uma parceria entre o Governo Estadual de São Paulo e uma instituição de Ensino Superior. Essa parceria resultou numa rede de formação docente que ofertou 1.000 vagas para o curso de especialização, com o objetivo de oferecer formação continuada e em serviço 
para professores do Ensino Fundamental e do Ensino Médio atuantes nas escolas da rede pública.

O curso, com duração de 12 meses (444 horas), foi dividido em 8 disciplinas. Destas, três se referiram à metodologia da pesquisa e não contemplaram OA em suas atividades. Assim, das cinco disciplinas que contemplaram OA, escolhemos a primeira e a última para analisar a acessibilidade, a saber: D01 - Diversidade e Cultura Inclusiva e D07 - Tecnologia Assistiva e Recursos de Apoio Pedagógico.

Na D01, o objeto educacional digital analisado chama-se "Diversidade e Cultura Inclusiva" e foi desenvolvido especialmente para essa disciplina. O objeto consiste em montar um quebra-cabeça diferente, como mostra a Imagem 1:

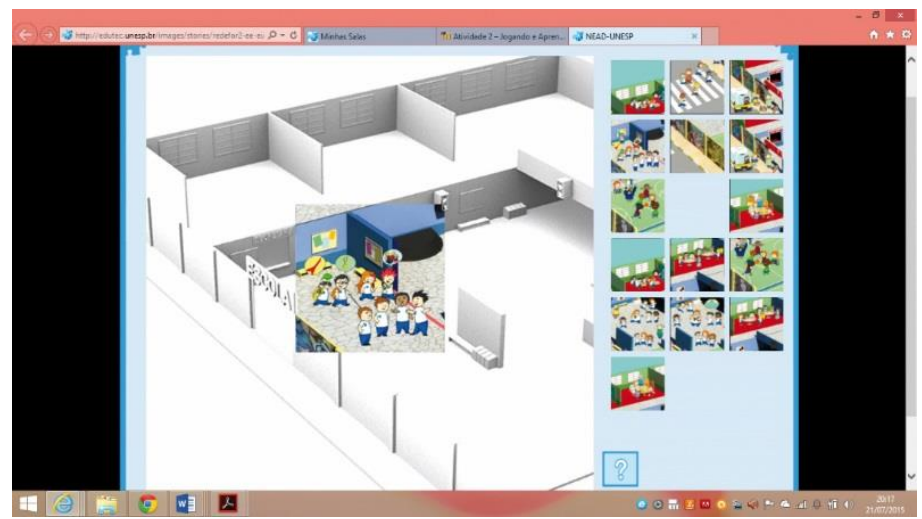

Imagem 1: Tela de jogo do OA Diversidade e Cultura Inclusiva.

O outro OA analisado chama-se "Estratégias de TA" e se refere à D07. Trata-se de um objeto que permite ao jogador escolher a imagem mais adequada ao enunciado proposto.

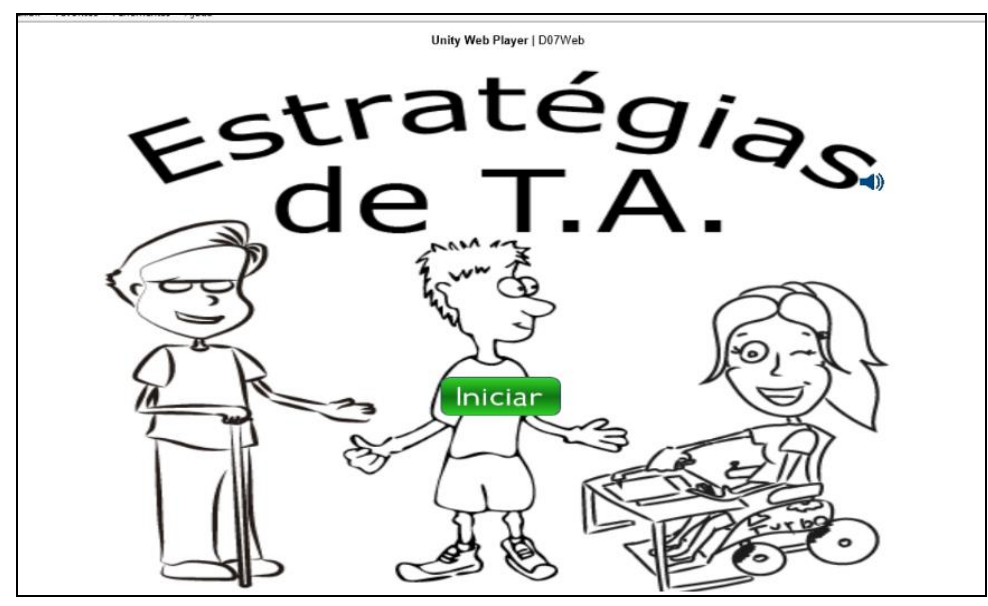

Imagem 2: Tela inicial do OA "Estratégias de TA". 
Sendo assim, mais especificamente dentro desse campo de pesquisa, tivemos como foco a análise de dois objetos de aprendizagem elaborados para essas duas disciplinas.

\subsection{Os procedimentos metodológicos}

Para a análise dos objetos de aprendizagem escolhidos para esta pesquisa tivemos como eixo norteador o objetivo deste estudo, a saber: examinar os objetos de aprendizagem acessíveis com base no produzido para duas disciplinas de um curso de especialização. Além disso, orientamo-nos a partir do seguinte questionamento: os OA disponíveis no AVA são acessíveis às pessoas que possuem necessidades especiais, como deficiência visual e auditiva?

Assim, para examinar esses OAs sob a ótica da acessibilidade, optamos por estabelecer categorias de análise que permitiram obter uma visão global dos recursos. São elas: Libras; Legendas: Audiodescrição e Navegação Simplificada. A categoria Libras e Legenda nos ajudaram a analisar a acessibilidade ao cursista surdo; a categoria Audiodescrição ao cursista cego; e a Navegação Simplificada para ambos.

Os OAs foram analisados qualitativamente, etapa a etapa de cada jogo, e os dados coletados organizados em uma planilha previamente elaborada para esse fim. Cada coluna corresponde a uma categoria; foram elaboradas questões norteadoras para auxiliar a análise, como no exemplo: Categoria Navegação Simplificada: a navegação pelo objeto é simples? É possível navegar utilizando apenas o teclado? É possível navegar fazendo movimentos simples com o mouse? Os botões têm tamanho suficiente para qualquer pessoa com necessidades especiais? Os links têm nomes claros?

Uma vez descritos os procedimentos metodológicos, apresentaremos a seguir os resultados obtidos. 


\section{Análise dos objetos de aprendizagem}

Com a intenção de facilitar o entendimento, apresentamos no Quadro 1 a síntese dos resultados deste estudo e na sequência as reflexões e discussões oriundas do processo de análise.

Quadro 1: Sistematização dos resultados da análise dos OAs.

\begin{tabular}{|l|c|c|}
\hline Categorias & OA1: "Diversidade e cultura inclusiva" & OA2: "Estratégias de TA" \\
\hline Libras & Não & Não \\
\hline Legendas & Não & Não \\
\hline Audiodescrição & Não & Sim \\
\hline $\begin{array}{l}\text { Navegação } \\
\text { Simplificada }\end{array}$ & Não & Sim \\
\hline
\end{tabular}

Assim como os ouvintes, os surdos são dotados de linguagem; entretanto, precisam de uma modalidade de língua para "ativar o seu potencial linguístico e, consequentemente, os outros potenciais, para que possam atuar na sociedade como cidadão atuante" (Scheffer et al., 2014, p. 316).

A Linguagem Brasileira de Sinais (Libras) é considerada a língua oficial dos surdos no Brasil, ou seja, sua linguagem natural. Por meio dela é possível a comunicação irrestrita do surdo, desde que o meio (ambiente físico ou virtual) esteja preparado para essa comunicação.

Com relação às pessoas cegas, não há como utilizar o mouse para acessar conteúdos por meio do computador. A audição e o tato são seus meios de comunicação e acesso à leitura das informações; para isso, fazem uso de atalhos do teclado para navegar pelos conteúdos (Leal Ferreira, 2008).

Diante disso, os OAs foram analisados de modo a verificar se são acessíveis às pessoas surdas, isto é, se possuem janela de Libras ou Legendas para que possam realizar as atividades de forma equitativa. Da mesma forma, se possuem audiodescrição para as pessoas cegas. Mas, como é possível observar no Quadro 1, nenhum dos dois OAs analisados apresenta tais itens de acessibilidade, com exceção da audiodescrição no OA2.

Entretanto, verificamos que ambos os objetos, mesmo não havendo janela em Libras, são acessíveis ao estudante surdo, uma vez que não há "falas" oralizadas. Ou 
seja, todas as instruções para o jogo estão escritas em língua portuguesa e, portanto, permitem ao surdo fazer a leitura dos comandos do jogo.

Por outro lado, o OA1 não demonstrou ser acessível ao estudante cego ou com baixa visão. As instruções são realizadas apenas na forma escrita; seria necessário que essas instruções fossem realizadas também na forma oral, como um leitor de tela. Além disso, considerando que o jogo se refere à montagem de um quebra-cabeça em que o jogador deve escolher a peça que mais se adéqua à abordagem inclusiva num ambiente escolar, seria necessária a audiodescrição de cada figura para que a pessoa cega ou com baixa visão pudesse selecionar e, assim, realizar a montagem do jogo.

A audiodescrição é uma atividade de mediação linguística, uma modalidade de tradução intersemiótica que transforma o visual em verbal, abrindo possibilidades maiores de acesso à cultura e à informação, contribuindo para a inclusão cultural, social e escolar. Além das pessoas com deficiência visual, a audiodescrição amplia o entendimento também de pessoas com deficiência intelectual, idosos e disléxicos (Motta, 2010, p. 2).

Todavia, pudemos observar que no OA2 há leitura de tela e instruções orais do início ao fim do jogo. Mas as imagens apresentadas nesse objeto não possuem audiodescrição, o que impossibilita a realização da atividade pelo cursista cego, pois ele não terá como escolher dentre as imagens a que corresponde ao enunciado, conforme a Imagem 3:

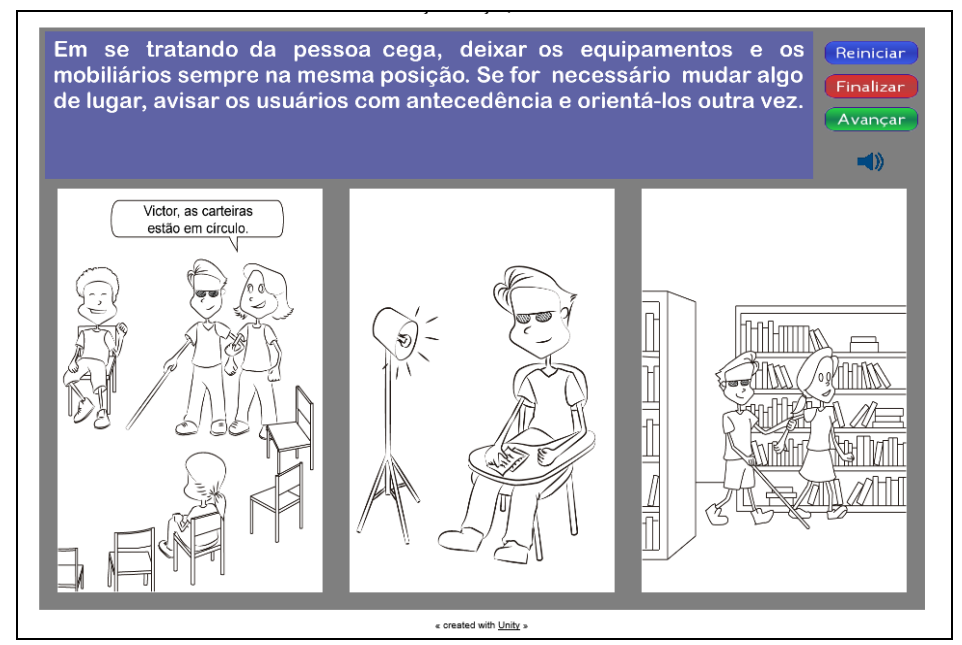

Imagem 3: Tela de atividade do OA2. 
Concordamos que a audiodescrição é recurso fundamental em ambos os OAs analisados, visto que há diversas imagens. Como já abordado, o OA1 também não apresenta audiodescrição, o que impossibilita o cego de realizar a atividade, mas funciona bem com o surdo, uma vez que não apresenta recursos sonoros.

Mesmo que o OA2 não apresente janela de Libras ou legenda, nós o consideramos acessível às pessoas surdas, pois, como no OA1, apresenta as instruções em língua portuguesa. Tanto o OA1 como o OA2 não apresentam vídeo ou personagem "falando" com o jogador; por isso, não verificamos a necessidade de janela em Libras ou legendas.

Quanto ao OA2, a navegabilidade é simples: usa-se a tecla 'Enter' para iniciar e a 'barra de espaço' para avançar, como mostra a imagem abaixo, retirada do objeto:

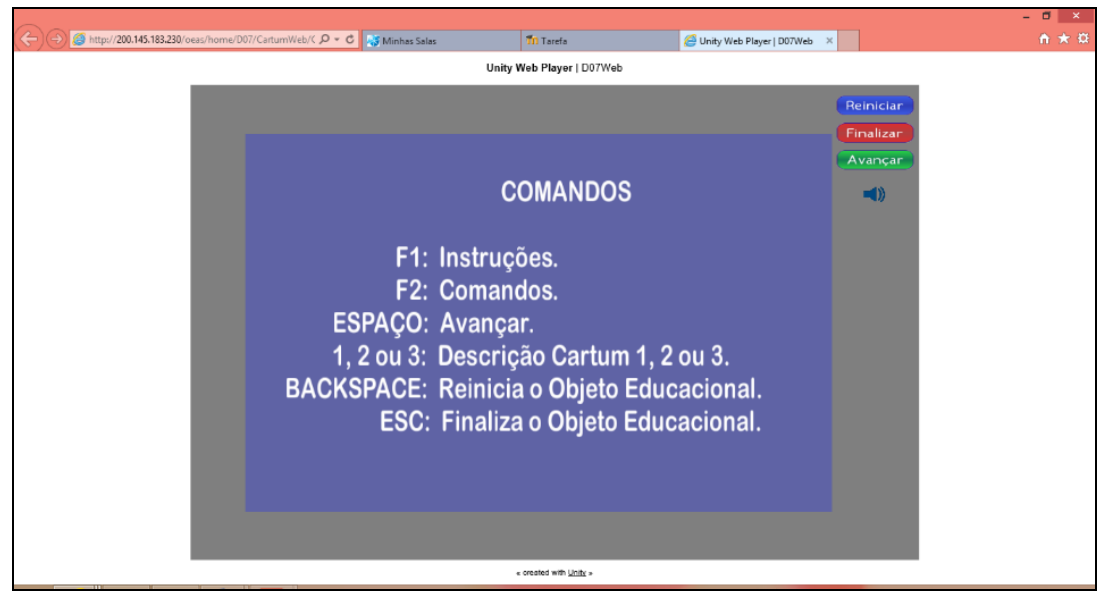

Imagem 4: Tela de navegação do OA2.

Dessa forma, o cursista surdo tem a possibilidade de realizar a atividade normalmente, pois tem instruções na tela na forma escrita em língua portuguesa. Como podemos verificar, o texto apresenta fundo de cor sólida e contrastante em uma única coluna para garantir a ordem de leitura e facilitar a compreensão.

No OA1, as instruções são claras e há a opção de navegar pelo objeto com leitores de tela, como do Dosvox ou Jaws, por exemplo. Entretanto, não há audiodescrição das imagens disponíveis para a realização da atividade. 


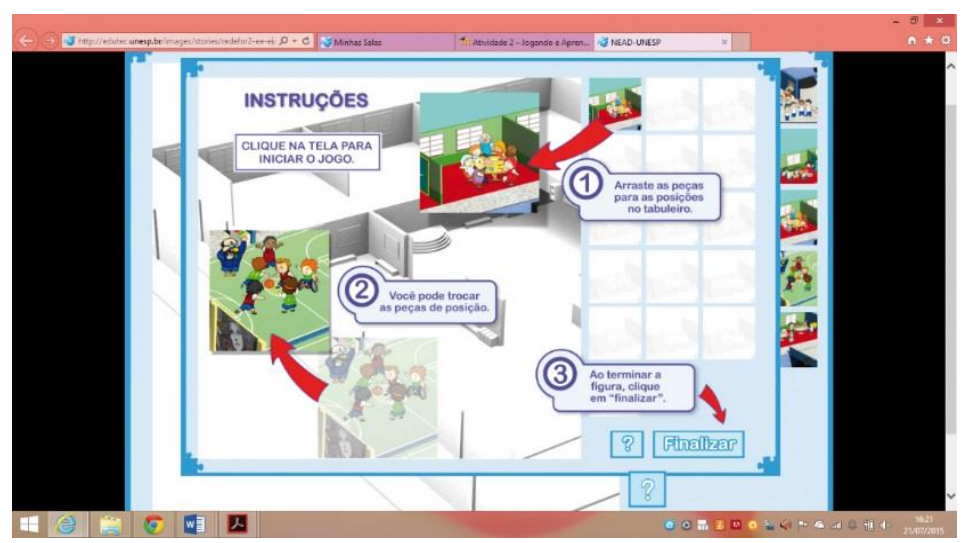

Imagem 5: tela de instrução do OA1.

O objeto não apresenta navegabilidade simples: não é possível navegar utilizando apenas o teclado. Os movimentos com o mouse devem ser o de 'arrastar' as imagens elencadas do lado direito ao centro da tela, o que é dificultoso para o cursista cego. As imagens são pequenas, impossibilitando a visualização com clareza de detalhes sem antes clicar em cima delas para aumentá-las, mesmo para as pessoas com visão preservada.

Diante de toda a análise realizada, baseando-se nas categorias estabelecidas para isso, como Libras, Legendas, Audiodescrição e Navegabilidade, os resultados evidenciaram que, embora haja esforço da universidade promotora do curso para oferecer acessibilidade em todo o AVA e materiais disponíveis, ainda são necessárias algumas adaptações nos OAs para que se tornem totalmente acessíveis.

Essa não é uma tarefa fácil e envolve um grande número de profissionais trabalhando em conjunto. Todavia, é por meio de iniciativas como essa que é possível o crescimento e o aprimoramento de objetos educacionais acessíveis para todos.

\section{Considerações finais}

A Educação a Distância é uma modalidade de ensino que vem crescendo vertiginosamente ano a ano, e sua expansão contribui de forma significativa para a democratização do ensino. Entretanto, essa democratização deve considerar o acesso e a permanência de todas as pessoas, independente do âmbito em que atua - presencial ou a distância. Assim, é necessário pensar em estratégias e metodologias que integrem a acessibilidade em ambientes virtuais para que aqueles que são privados de algum 
sentido, como a visão e a audição, possam ter participação equitativa em cursos a distância.

Tendo como foco os princípios da inclusão e fundamentação em documentos oficiais e legislação, que apontam a educação como um direito de todos, propomo-nos a verificar se os OAs disponíveis no ambiente virtual de aprendizagem (AVA) são acessíveis às pessoas que possuem necessidades especiais, como deficiência visual e auditiva.

Assim, este trabalho teve como objetivo analisar objetos de aprendizagem oferecidos em duas disciplinas de um curso de especialização a distância. O curso foi oferecido a professores em exercício da rede pública estadual.

Por meio de revisão bibliográfica e estudo de caso, analisamos a acessibilidade em dois OAs. Cada etapa dos jogos foi analisada com base em categorias preestabelecidas: Audiodescrição, Libras, Legendas e Navegação Simplificada.

O estudo revelou a complexidade na promoção de recursos acessíveis em AVA. Nenhum dos OAs analisados demonstrou estar totalmente acessível às pessoas cegas e/ou surdas. Todavia, ambos poderiam ser utilizados pelas pessoas surdas, por não terem falas "oralizadas" ou recursos sonoros, o que permite ao surdo ler os comandos e realizar as atividades propostas. Para as pessoas cegas, isso não seria possível, uma vez que os recursos analisados não demonstraram acessibilidade a esse público.

O principal questionamento que impulsionou esta pesquisa foi se os OAs oferecidos no curso estavam acessíveis às pessoas com necessidades especiais. De maneira geral, chegamos à conclusão de que não estão totalmente acessíveis, embora as pessoas surdas tivessem condições de manuseá-los.

Após a conclusão deste trabalho, além desse questionamento inicial, é possível apontar outros que podem se tornar objeto de estudo de futuras investigações, como: de quais estratégias o tutor poderá lançar mão para auxiliar os cursistas com necessidades especiais na execução de um curso a distância, principalmente na mediação em fóruns de discussão e atividades coletivas? Como tornar um AVA e um OA acessíveis? O que levar em consideração e/ou como elaborar um curso acessível?

Este estudo também concluiu que a instituição de ensino superior promotora do curso tem se empenhado em oferecer acessibilidade, e podemos considerá-la uma das 
pioneiras em iniciativas como essa. Isso porque, embora os OAs não estejam totalmente acessíveis, outros recursos como vídeos e e-books já contam com legendas e Libras.

Esperamos que as reflexões oriundas desta investigação possam promover mais discussões sobre o tema e contribuam com a inclusão das pessoas com necessidades especiais nos níveis mais elevados da escolarização, por meio da EaD, e impulsionem a qualidade da educação para todos em nosso país.

\section{Agradecimentos}

Agradecemos à coordenação do Curso de Especialização em Educação Especial na Perspectiva da Educação Inclusiva da Universidade Estadual Paulista "Júlio de Mesquita Filho" (Unesp), Núcleo de Educação a Distância (NEaD), pela oportunidade da realização desta pesquisa.

\section{Referências bibliográficas}

Audino, D. F., \& Nascimento, R. da S. (2010). Objetos de aprendizagem: Diálogo entre conceitos e uma nova proposição aplicada à educação. Revista Contemporânea de Educação, 5(10), 128-148. Disponível em http://revistas.ufrj.br/index.php/rce/article/view/1620/1468

Beck, R. J. (2001). Learning objects: what?. Center for International Education. University of Wisconsin. Milwaukee.

Brasil. (1994). Declaração de Salamanca e linha de ação sobre necessidades educativas especiais. Brasília: Unesco.

Leal Ferreira, S. B., \& Nunes, R. R. (2008). e-Usabilidade. Rio de Janeiro: LTC.

Macedo, C. M. S. (2013). Diretrizes de acessibilidade em conteúdos didáticos. Revista Brasileira de Design da Informação, 10(2), 123-136. Disponível em http://www.infodesign.org.br/infodesign/article/download/193/136

Maciel, C., \& Backes, E. M. (2012). Objetos de aprendizagem, objetos educacionais, repositórios e critérios para a sua avaliação. In: MACIEL, C. (org.). Ambientes 
virtuais de aprendizagem. Cuiabá: Editora da UFMT, 161-198. Disponível em http://disciplinas.stoa.usp.br/pluginfile.php/129865/mod resource/content/1/A mbientes\%20Virtuais.pdf

Motta, L. M. V. M., \& Romeu Filho, P. (org.). (2010). Audiodescrição: transformando imagens em palavras. São Paulo: Secretaria dos Direitos da Pessoa com Deficiência. Disponível em http://www.vercompalavras.com.br Ldownload/audiodescricao-transformando-imagens-em-palavras.pdf

Organização das Nações Unidas (ONU). (2006). Convenção sobre os Direitos das Pessoas com Deficiência.

Sabbatini, M. (2012). Reflexões críticas sobre o conceito de objeto de aprendizagem aplicado ao ensino de Ciências e Matemática. Revista de Educação Matemática e Tecnológica Ibero-americana, 3(3). Disponível em http://www.gente.eti.br/revistas/index.php/emteia/article/view/103

Sampaio, R. L., \& Almeida, A. R. S. (2010). Aprendendo Matemática com objetos de aprendizagem. Ciências \& Cognição, 15(1), 64-75. Disponível em http://www.cienciasecognicao.org/revista/index.php/cec/article/view/290

Scheffer, M. L. C., Bez, M. R., \& Passerino, L. M. (2014). Mídias digitais na educação dos surdos. In: Tarouco, L. M. R., Ávila, B. G., Santos, E. F. dos, Bez, M. R., \& Costa, V. (orgs.). Objetos de aprendizagem: teoria e prática. Porto Alegre: Evangraf.

Spinelli, W. Os objetos virtuais de aprendizagem: ação, criação e conhecimento. Disponível em http://www.lapef.fe.usp.br/rived/textoscomplementares ttextoImodulo5.pdf

Unesp. (2014). Tecnologias na Educação Superior: ambientes virtuais e materiais didáticos digitais acessíveis na Unesp. São Paulo: Cultura Acadêmica/Universidade Estadual Paulista/Núcleo de Educação a Distância.

Wiley, D. (2001). Connecting learning objects to instructional design theory: a definition, a metaphor and taxonomy. Disponível em www.reusability.org/read/chapters/wiley.doc 